\title{
FREQUENCY OF DISLIPIDEMIA.
}

1. MBBS, FCPS (Medicine)

Assistant Professor, PMC, FSD.

2. MBBS, FCPS Medicine,

FCPS Gastroenterology,

MRCP (Medicine), SCFC (Gastro)

Assistant Professor, PMC, FSD

3. MBBS, FCPS (Medicine)

Associate Professor, PMC, FSD.

4. MBBS, FCPS (Medicine)

Assistant Professor, PMC, FSD.

Correspondence Address:

Dr. Muhammad Adrees Shani

Assistant Professor, PMC, FSD.

haroonadrees2010@gmail.com

Article received on:

15/04/2017

Accepted for publication:

05/10/2017

Received after proof reading: 29/11/2017
Muhammad Adrees Shani ${ }^{1}$, Hafiz Mughees Ather ${ }^{2}$, Muhammad Hanif Nagra ${ }^{3}$, Muhammad Aamer ${ }^{4}$

ABSTRACT... Background: Lipid lowering therapy in documented Coronary artery disease is considered secondary prevention and lipid levels have significant influence on CAD Death rates. Objectives: To find out the frequency of dyslipidemia in patients of ischemic heart disease patients and to compare the results with international studies. Study Design: A descriptive case series study was carried out. Setting: The study was done in Medical Unit-II and CCU of Allied Hospital Faisalabad. For 6 months from 15march 2016 to 15 September 2016. Subjects: 50 patients diagnosed to have angina pectoris and ischemic heart disease patients were included. and all 50 Patients were on routine diet and were asked not to take anything overnight. Blood sample (5cc) was taken in all 50 patients with overnight fasting and blood sample were sent to Allied Hospital Laboratory immediately for analysis of (Triglycerides, total cholesterol, LDL and HDL). Reagents used were those of: "Merck Diagnostia Germany" Triglycerides were measured by ready to use reagent-using enzymatic splitting with lipoprotein lipase and quinoneimine as indicator. Total cholesterol, HDL and LDL were measured by "CHOD PAP METHOD" using ready to use reagents. Methods: Diagnosis was made on the basis of history, clinical examination and ECG findings. The history included chest pain, palpitation, sweating and shortness of breath. Ischemic heart disease patients'. ECG criteria were ST segment depression, T-wave flattening and inversion. All patients were subjected to fasting lipid profile. Results: Out of 50 patients, 30 were males and 20 were females. The frequency of dyslipidemia was $60 \%$ in patients of angina pectoris and ischemic heart disease patients. Triglycerides were elevated in $56 \%$. Male were $40 \%$ and females $16 \%$ and $P$ value regarding gender distribution was 0.063 ; significant one indicating that there is a definitive relation between gender and hypertriglyceridemia. Total cholesterol was elevated in $20 \%$. Males were $12 \%$ and females were $8 \%$. And $\mathrm{P}$ value regarding sex distribution was 1.00. LDL was elevated in only $4 \%$. HDL was also elevated in $4 \%$ of the cases. Conclusion: Present study concluded that dyslipidemia is common in angina pectoris and ischemic heart disease patients especially males and all patients of angina pectoris must be screened for dyslipidemia as early as possible and should be treated aggressively to minimize the risk of further complication of angina pectoris.

Key words: $\quad$ Dislipidemia, Ischemic Heart Disease.

Article Citation: Shani MA, Ather HM, Nagra MH, Aamer M. Frequency of duslipidemia. Professional Med J 2017;24(12):1828-1834. DOI:10.17957/TPMJ/17.3981

\section{INTRODUCTION}

More and more concerns of the world about prevention of diseases rather than treatment because it cost effective and also improve the expectancy of life. different risk factors which are reversible in diseases are identified as early as possible to decrease the mortality and morbidity. Dyslipidemia is one of the important reversible risk factor for ischemic heart disease patients.

Lipids are synthesized in liver and intestine and they are transported to various tissues for their metabolic function in the form of lipoprotein, like chylomicron LDL cholesterol, total cholesterol, triglycerides and HDL cholesterol. Cardiovascular diseases and dyslipidemia is strongly associated with high mortality and morbidity. Main clinical consequence of dyslipidemia is premature atherosclerosis.

In atherosclerosis there is accumulation of lipids in arteries and lead to clinical manifestation of angina or myocardial infarction depending on partial occlusion or complete occlusion of coronary arteries. 
In Different studies there is clear consensus that elevated LDL cholesterol and low HDL are associated with increased risk of atherosclerosis.

Angina pectoris is clinical symptoms of ischemic heart disease. Angina limits the daily normal activities and has negative impact on quality of life even there has been evidence that 1 year after coronary revascularization one third of patients are not able to return to work in USA so it isnotonly effect quality of life of individual but also impact on economy of individual. There is definitive evidence that early recognition of dyslipidemia and early use of stations have marked reduced the mortality and morbidity in cardiovascular patients. For this reason this study was conducted to see the frequency of dyslipidemia in ischemic heart patients.

\section{AIMS AND OBJECTIVES}

The objectives of my study were:

1. To find out the frequency of dyslipidemia in patients of angina pectoris and ischemic heart disease patients

2. To compare the results with international studies.

\section{MATERIAL AND METHODS}

\section{Setting}

My study was carried out in Medical Unit-2 and CCU of Allied Hospital Faisalabad, Pakistan.

\section{Duration}

My study was carried out for six months from 15 March 2016 to 15 September 2016.

\section{Sample Size}

A study of 50 cases on patients of angina pectoris was carried out.

\section{Sampling Technique}

The sampling of my study was by simple random (probability).

\section{Study Design}

A descriptive case series study of 50 patients of angina pectoris and ischemic heart disease patients was carried out in Medical Unit-II and
CCU of Allied Hospital Faisalabad.

\section{Inclusion Criteria}

1. All patients of either sex with stable and unstable angina and ischemic heart patient.

2. Age between 25 to 70 years.

\section{Exclusion Criteria}

1. Patients having angina pectoris diagnosed on ETT only.

2. Patients with dilated cardiomyopathies

3. Patients with Valvular heart disease

\section{Data Collection Procedure}

This study was conducted on 50 patients (25 to 70 years of age admitted in Medical Unit-2 and CCU of Allied Hospital Faisalabad with a provisional diagnosis of angina pectoris on the basis of history, clinical examination and ECG findings) were screened for dyslipidemia.

Patients were properly informed about the study and consent was taken.

\section{History}

History was taken in 50 patients and following was included in history, Chest pain, palpitation, sweating, and shortness of breath and associated symptoms.

\section{Clinical Examination}

Clinical examination was done on each patient of angina pectoris and it included general physical examination, cardiovascular system, respiratory system, central nervous system and gastrointestinal system examination.

\section{Investigation}

ECG was done in every patient and ECG machine used was Kenz. ECG 108 Class 1, Model 00020345 (Single Channel) and twelve leads ECG was done and ECG criteria for diagnosis of angina pectoris were ST segment depression, $\mathrm{T}$ wave changes include $\mathrm{T}$ wave flattening and inversion.

\section{Fasting Lipid Profile}

All 50 Patients were on routine diet, asked not to take anything overnight. Blood sample (5cc) was taken in all 50 patients with overnight 
fasting and blood sample were sent to Allied Hospital Laboratory immediately for analysis of (Triglycerides, total cholesterol, LDL and HDL).

Reagents used were those of: "Merck Diagnostia Germany" Triglycerides were measured by ready to use reagent-using enzymatic splitting with lipoprotein lipase and quinoneimine as indicator.

Total cholesterol, HDL and LDL were measured by "CHOD PAP METHOD" using ready to use reagents.

Blood complete examination was also done in every patient.

\section{Statistical Analysis}

Data was analyzed by using chi-square test and statistical analysis was carried out with the use of SPSS Version 20 for window 7.P values were calculated.

\section{RESULTS}

This study was carried out on 50 patients of angina pectoris and ischemic heart disease patients. Diagnosis was made on the basis of history, clinical examination and ECG findings. All the patients were subjected to fasting lipid profile.

Out of 50 patients, 30 were male and 20 were females as shown in the pie Figure-1. Mean age of whole population under study was 56.20 \pm 10.1 years. Mean age of male patients was $56.77 \pm 10.7$ years. Mean age of females was $55.35 \pm 9.5$ years. Distribution of Dislipdemia in angina patients according to gender in Figure-1.

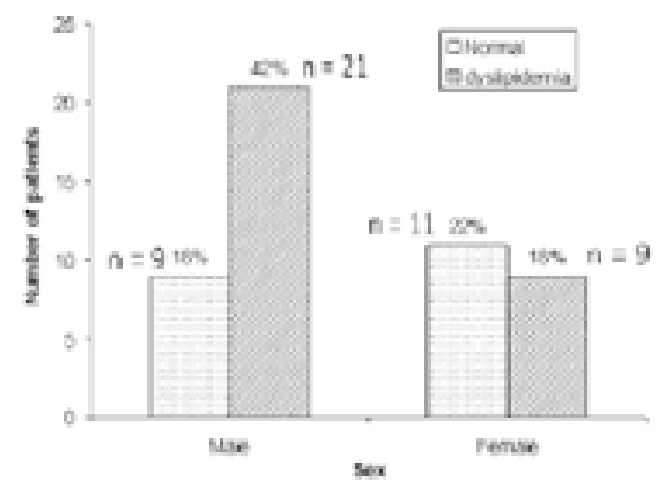

Figure-1. Distribution of Angina Pectoris patients according to dyslipidemia and Gender
These patients were arranged in four groups as under and shown in Figure-2:

Group I< 40 years

Group II40 to 50 years

Group III50 to 60 years

Group IV > 60 years

Majority of them fall in Group II, III, IV (about $92 \%$ ) of the total patients indicating that angina pectoris is more common in this age group.

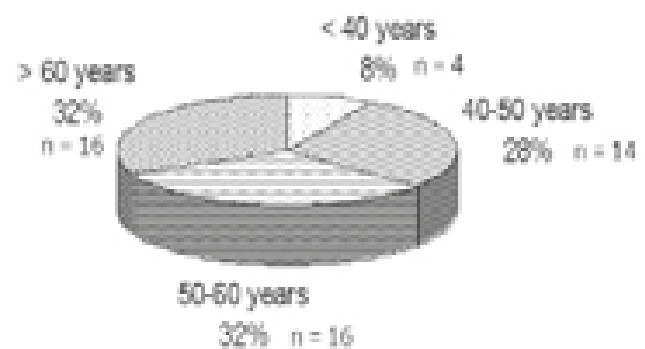

Figure-2. Percentage of different age groups has been shown.

The frequency of dyslipidemia was $60 \%$ in patients of angina pectoris as a whole shown in Figure-3.

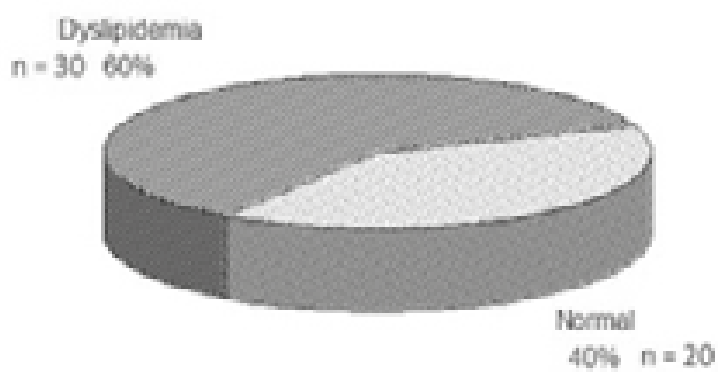

Figure-3. Distribution of angina pectoris patients and IHD pts according to dyslipidemia.

Triglycerides were elevated in $56 \%(n=28)$ of the total patients and out of $56 \%$ patients, $40 \%(n=20)$ were male and $16 \%(n=8)$ were females and $P$ value regarding sex distribution was 0.063 , significant one indicating that there is a definitive relation between sex and hypertriglyceridemia shown in Table-I.

Intergroup analysis of variance regarding age and triglycerides showed $P$ value 0.0796 , significant one indicating that there is a definitive relation between age and hypertriglyceridemia and maximum level was found to be $428 \mathrm{mg} / \mathrm{dl}$. 
Patient was hypertensive male shown in Table-II.

Total cholesterol was elevated in $20 \%(n=10)$ of the total patients and out of $20 \%$. Males were $12 \%$ and females were $8 \%$. And $\mathrm{P}$ value regarding sex was 1.00 indicating that there is no sex discrimination regarding total cholesterol.
Intergroup analysis of variance regarding age and total cholesterol showed $P$ value of 0.416 indicating that there is no significant relationship between age and total cholesterol.

Highest value was found in a female of 45 years old poor fellow.

\begin{tabular}{|c|c|c|c|c|c|c|}
\hline \multirow{3}{*}{ Sex } & \multicolumn{4}{|c|}{ Triglycerides } & \multirow[b]{3}{*}{ No. } & \multirow[b]{3}{*}{$\%$} \\
\hline & \multicolumn{2}{|c|}{ < 150 (normal) } & \multicolumn{2}{|c|}{$>150$} & & \\
\hline & No. & $\%$ & No. & $\%$ & & \\
\hline Female & 12 & 24.0 & 8 & 16.0 & 20 & 40 \\
\hline Total & 22 & 44.0 & 28 & 56.0 & 50 & 100 \\
\hline
\end{tabular}

\begin{tabular}{|c|c|c|c|c|c|c|}
\hline \multirow{3}{*}{ Age group } & \multicolumn{4}{|c|}{ Triglycerides } & \multirow{2}{*}{\multicolumn{2}{|c|}{ Total }} \\
\hline & \multicolumn{2}{|c|}{$<150$ (normal) } & \multicolumn{2}{|c|}{$>150$} & & \\
\hline & No. & $\%$ & No. & $\%$ & No. & $\%$ \\
\hline$<40$ years & 2 & 4.0 & 2 & 4.0 & 4 & 8.0 \\
\hline $40-50$ years & 5 & 10.0 & 9 & 18.0 & 14 & 28.0 \\
\hline $50-60$ years & 4 & 8.0 & 12 & 24.0 & 16 & 32.0 \\
\hline$>60$ years & 11 & 22.0 & 5 & 10.0 & 16 & 32.0 \\
\hline
\end{tabular}

LDL was elevated in only $4 \%$ of total patients. $2 \%$ were males and $2 \%$ were females. $P$ value regarding sex distribution was 0.7683 (nonsignificant).

Intergroup analysis of variance regarding age and LDL showed $P$ value of 0.0984 , significant one indicating that there is a definitive relation between age and LDL.

HDL was elevated in only $4 \%$ of total patients. $2 \%$ were males and $2 \%$ were females. $P$ value regarding sex distribution was 0.7683 nonsignificant.

Intergroup analysis of variance regarding age and $\mathrm{HDL}$ showed $\mathrm{P}$ value of 0.7598 indicating that there is no definitive relation between age and HDL.

Chi square test application to the two variables i.e. Lipids and Angina pectoris showed that: -

Dyslipidemiain angina pectoris patients showed a $P$ value of 0.0206 statistically significant indicating that there is a definitive relation between angina pectoris and dyslipidemia.

Triglycerides statistically are significantly related to the age group with $P$ value of 0.0796 and Triglycerides are commonly raised in age between 40 to 60 years $(42 \%(n=21)$ out of $56 \%(n=28)$ of total patients who have hypertriglyceridemia) Triglycerides are also significantly related to sex showing $P$ value of 0.063 . So Triglycerides are more commonly raised in males $40 \% \quad(n=20)$ as compared to females $18 \%(n=9)$ in our study. Triglycerides are also significantly related to total cholesterol with a $P$ value of 0.039 showing that triglycerides have significant relation with total cholesterol.

Total cholesterol and LDL are statistically related to each other with a $P$ value of 0.047 .

LDL is significantly related to positive family history of ischemic heart disease with a $P$ value of 0.0254 .

HDL is significantly related to diabetes Mellitus 
with a $P$ value of 0.0771 shown in Table-III.

$\mathrm{HDL}$ is also significantly related to socioeconomic group of middle class with a $P$ value of 0.206 .

\begin{tabular}{|c|c|c|c|c|c|c|}
\hline \multirow{3}{*}{$\begin{array}{l}\text { Diabetes } \\
\text { mellitus }\end{array}$} & \multicolumn{4}{|c|}{ HDL (mg/dl) } & \multirow{2}{*}{\multicolumn{2}{|c|}{ Total }} \\
\hline & \multicolumn{2}{|c|}{$<35$} & \multicolumn{2}{|c|}{ > 35 (normal) } & & \\
\hline & No. & $\%$ & No. & $\%$ & No. & $\%$ \\
\hline No & 0 & 0.0 & 30 & 60.0 & 30 & 60 \\
\hline Yes & 2 & 4.0 & 18 & 36.0 & 20 & 40 \\
\hline Total & 2 & 4.0 & 48 & 96.0 & 50 & 100 \\
\hline
\end{tabular}

\section{DISCUSSION}

Advancement in the medical science and other technology has resulted in early detection of the diseases and risk factors prolonging the survival of the patients suffering from cardiovascular diseases.

More and more is being known about the pathogenesis and risk factors, their role in progression of the disease. Researches have been going on to reduce these risk factors as early as possible in the disease to prolong the life of the patients.

Angina pectoris have multiple risk factors like DM, Hypertension, Smoking, Dyslipidemia, positive Family history, Male gender, Obesity and Postmenopausal.

Lipids abnormalities are very common in angina pectoris and other ischemic heart disease patients. It is one of the reversible risk factor, which can be detected very early in patients who have positive family history of ischemic heart disease and in other patients as well so that we can minimize the risk of developing angina or we can decrease the progression of the disease.

Present study is also concerned with the frequency of dyslipidemia in angina patient 50 patients were included in this study and 30 were male patients $(60 \%)$ and 20 were female patient $(40 \%)$ and majority of our patients were in age between 40 to 70 years. This shows that angina pectoris is more common in this age group. Dyslipidemia was found in $60 \%$ of the total patients and hypertriglyceridemia was found in $56 \%$ of the patients.
Andrikopoulos G1, Tzeis S, Mantas I, Olympios $\mathrm{C}$, and his colleagues carried out study on ischemic heart disease patients at Greece and it was published in Hellenic J Cardiol. 2012 Jan-Feb. Total of 418 consecutive patients with ACS $(78.0 \%$ males, $63.9 \pm 12.9$ years) from 17 centers in Greece were enrolled in this study. Three hundred and forty patients (male 78\%) were enrolled in this study. Mean age in this study was $63.9+/ 12.9$ years. Following data was collected from these patients. ${ }^{6}$ Dyslipidemia was the most common risk factor in these patients $(57.4 \%)$ followed by hypertension $(67.9 \%)$ and diabetes in $(27.5 \%)$ which is similar to our study which showed dyslipidemia in $60 \%$ of the cases and hypertension was also found in $50 \%$ of the patients and $40 \%$ of were also diabetic. ${ }^{5}$

Pećin I1, Milicić D, Jurin $H$, Reiner $Z$, and his colleagues conducted another study in Croatia. ${ }^{8}$ This was published in CollAntropol. 2012 Jun. They analyzed the value of the clinical data in ischemic heart disease patients. 122 patients were followed prospectively male (88), women (34) with age $66.3+/$. Clinical evaluation was done between 2003 and 2010. Their vascular risk factors were analyzed. The most frequent risk factors were dyslipidemia in $(90 \%)$ of the cases, which is different from our study with that showed dyslipidemia in $60 \%$ of the patients. Hypertension in $95 \%$ patients compared to $50 \%$ in our study and diabetic $25 \%$ compare to our study $27.5 \%$. The difference between $90 \%$ dyslipidemia in this study and $60 \%$ dyslipidemia in our study may be due to because more number of Hypertensive cases were included in this study and there may be regional difference of risk factors in Croatia and Pakistani population need further study for 
this difference. ${ }^{6}$

In Pakistan local study conducted at BahawalpurVictoria Hospital in Bahawalpur by Alamgir M A, Fayyaz M, Jamil A, Sharif Nand his colleagues for the analysis of pattern of Dyslipidemia amongst patient with diabetes mellitus with cardiovascular disease.

Risk factor analysis of ischemic heart disease in patients presenting for angiographies between July 2008 to December 2008 on 100 patients of diabetes mellitus with cardiovascular disease. ${ }^{9}$ Majority of patients presented in middle and old age category. High percentage of complications were observed in patients of middle age group with great frequency of cardiovascular complications. ${ }^{7}$

LDL - C concentration was high normal (188 \pm $23.4 \mathrm{mg} / \mathrm{dl}$ ) in these cases. Collectively the mean concentration of LDL-C was raised and statistically very highly significant in all these macro vascular complications validating our study as well.

Khattab AA1, Knecht M, Meier B, Windecker S, $I$ and his colleague conducted another study in patients with uncontrolled cardiovascular risk factor with stable coronary artery disease was published in Euro J PrevCardiol. 2013 Oct. ${ }^{4}$

Patients' population was 207 and 13 month follow up was done .Risk factor analysis showed LDL levels increased in $47.2 \%$ which is different to our study and difference between $4 \%$ and $47.2 \%$ may be regional difference European and Pakistani population .

Secondly population in European study these patients were not doing regular exercise and were not advised proper dietary advice and only $8 \%$ had quit smoking that may be reason for \% age difference between our study and European study.

In another study conducted at Vietnam in Thong Nhat HOSPITAL at HO CHIN minh city. In this study 338 patients were enrolled and study was done from 2009 to 2011 and it was published IN J atherosclerthromb IN 2014.

Prevalence of dyslipidemia in nonelderly patients was $56.9 \%$ of patients which is similar to our study with dyslipidemia in $60 \%$ patients validating our study

\section{CONCLUSION}

Following conclusion can be made from this study;

- Frequency of dyslipidemia in angina pectoris patients is $60 \%$

- Dyslipidemia is two times more common in males than females.

- Dyslipidemia commonly occurs in angina pectoris patients.

- Reduced HDL is more common in angina pectoris patients who are diabetic as well although it occurs in a very lesser percentage as compared to triglycerides and total cholesterol.

- It is therefore recommended that;

- All patients of angina pectoris should be screened for dyslipidemia as early as possible and should be treated aggressively to minimize the risk of further complication of angina pectoris.

- All angina patients should be educated about the importance of controlling the dyslipidemia to prevent the complication. And regular follow up and monitoring of lipid profile of the patients should be done.

\section{Copyright $@ 05$ Oct, 2017.}

\section{REFERENCES}

1. Summerton C, Shetty P, Sandle L N, Watts S, Nutritional, metabolic and environmental disease. In: Haslett C, Chilver E R, Boon N A, Colledge, N R, John A, Hunter. Editors. Davidson's Principles of and Practice of Medicine, 19th edi London Churchill Livingston 2002; 308.

2. Gale EAM, Anderson JV, Diabetes mellitus and other disorders of metabolism. In: Kumar P, Clark M, editors. Kumar and Clark Clinical Medicine. 8th edi Edinburgh W B Saunders 2012; 1033.

3. Andrew P, Selwyn, Braunwald E, Cardiovascular disease. In: Braunwald E, Fuaci AS, Kasper D L, Stephen L, Hauser SL, Longo D L, Jameson L, editors. Harrison's Principles of Medicine 19thedi New York. McGraw-Hill, 2015:2435. 
4. Khattab AA1, Knecht M, Meier B, Windecker S, Schmid JP, Wilhelm M, Saner $H$. Persistence of uncontrolled cardiovascular risk factors in patients treated with percutaneous interventions for stable coronary artery disease not receiving cardiac rehabilitation. Eur J PrevCardiol. 2013 Oct; 20(5):743-9. doi: 10.1177/2047487312447751. Epub 2012 Apr 30.

5. Andrikopoulos G1, Tzeis S, Mantas I, Olympios C, Kitsiou A, Kartalis A, Kranidis A, Tsaknakis T, Richter D, Pras A, Pipilis A, Lampropoulos S, Oikonomou K, Gotsis A, Anastasiou-Nana M, Triposkiadis F, Goudevenos J, Theodorakis G, Vardas P. Epidemiological characteristics and in-hospital management of acute coronary syndrome patients in Greece: results from the TARGET study. Hellenic J Cardiol. 2012 Jan-Feb; 53(1):33-40.

6. Pećin I1, Milicić D, Jurin H, Reiner Z. A pilot Croatian survey of risk factor (CRO-SURF) management in patients with cardiovascular disease. CollAntropol. 2012 Jun; 36(2):369-73.

7. Alamgir M A, Fayyaz M, Jamil A, Sharif N. To determine the patterns of dyslipidemia amongst the macro vascular complications of type II diabetes mellitus. Ann King Edward Med Uni Jul - Sep 2010; 16(3):169-73.

8. Shafique Ahmad, Shehzad Ahmad, Mohammad Kashif. Frequency of Modifiable Risk Factors at the Time of Myocardial Infarction and at Twelve Months Follow Up.Pak J Med Health Sci Jul - Sep 2012; 6(3):779-82.

9. Duc Cong N1, Dung HT. The risk factors of acute coronary syndrome in patients over 65 years old at Thong Nhat Hospital of Ho Chi Minh City, Vietnam. J AtherosclerThromb. 2014; 21Suppl 1:S36-41.

10. M.N. Krishnan. Coronary heart disease and risk factors in India - On the brink of an epidemiclndian Heart J. 2012 Jul; 64(4): 364-367.doi: 10.1016/j. ihj.2012.07.001.

11. BMC CardiovascDisord. 2016 Jan 14; 16:13. doi: 10.1186/s12872-016-0190-x. Korneva VA, KyznetsovaTlu, Mandel'shtamMlu, Konstantinov VO, Vasil'ev VB. [The clinical manifestations of atherosclerosis in familial hypercholesterolemia]. TerArkh. 2014; 86(1):18-22.

12. Silbiger JJ1, Stein R, Roy M, Nair MK, Cohen P, Shaffer J, Pinkhasov A, Kamran M. Coronary artery disease in South Asian immigrants living in New York City: angiographic findings and risk factor burdensEthn Dis. 2013 Summer; 23(3):292-5.

13. S. Khadke, ShubhangiHarke, A. Ghadge, O. Kulkarni, SupriyaBhalerao, Arundhati, Diwan et al. Association of Fasting Plasma Glucose and Serum Lipids in Type 2. Diabetics. Indian J Pharm Sci. 2015 Sep-Oct; 77(5): $630-634$.

14. Manish Gutch, Syed MohdRazi, Sukriti Kumar and Keshav Kumar Gupta. Diabetes mellitus: Trends in northern India. Indian J EndocrinolMetab. 2014 SepOct; 18(5): 731-734.

15. Syed Muhammad Shahid, SyedaNuzhatNawab, Rozeena Shaikh and Tabassum Mahboob. Glycemic control, dyslipidemia and endothelial dysfunction in coexisted diabetes, hypertension and nephropathy. Pak J Pharm Sci Jan - Jan 2012;25(1):123-9.

16. Muhammad Asif Bhalli, Azhar Mehmood Kayani and Naseer Ahmed Samore. Frequency of Risk Factors in Male Patients with Acute Coronary Syndrome. JCPSP 2011, Vol. 21 (5): 271-275.

\begin{tabular}{|c|l|l|}
\hline \multicolumn{3}{|c|}{ AUTHORSHIP AND CONTRIBUTION DECLARATION } \\
\hline Sr. \# & \multicolumn{1}{|c|}{ Author-s Full Name } & \multicolumn{1}{|c|}{ Contribution to the paper } \\
\hline 1 & M. Adrees Shani & Data collection \\
\hline 2 & Hafiz Mughees Ather & Review manuscript \\
\hline 3 & M. Hanif Nagra & Manuscript writing \\
\hline 4 & Muhammad Aamer & Manuscript writing \\
\hline
\end{tabular}

